\title{
Successful fluconazole treatment of catheter-related candidemia caused by Candida haemulonii: report of 2 neonatal cases
}

\author{
Fang-Qin Xia, M.D. ${ }^{a}$, Chu-Yuan Ye, M.D. ${ }^{a}$, Wei-Wei Xie, M.D. ${ }^{a}$, Neha-Devi Poonit, M.D. ${ }^{a}$ \\ and Yong-Hai, Zhou, M.D. ${ }^{a}$
}

\begin{abstract}
Candida haemulonii forms part of the non-albicans Candida species. The candidemia caused by $C$. haemulonii is extremely rare but fatal in neonates. We reported the first two neonates with C. haemulonii candidemia in China which were treated with fluconazole and reviewed two papers previously reported. Our report adds further awareness on C. haemulonii candidemia in critical neonates and points out the importance of an early diagnosis and treatment of this fatal infection.

Key words: neonate; Candida haemulonii; candidemia.
\end{abstract}

http: / / dx.doi.org/10.5546/ aap.2018.eng.e663

To cite: Xia FQ, Ye CY, Xie WW, et al. Successful fluconazole treatment of catheter-related candidemia caused by Candida haemulonii: report of 2 neonatal cases. Arch Argent Pediatr 2018;116(5):e663-e666.

\section{BACKGROUND}

Candida haemulonii, a non-albicans Candida species, was first isolated from a human in 1984. ${ }^{1}$ The candidemia caused by C. haemulonii is extremely rare but fatal in the Neonatal Intensive Care Unit (NICU). ${ }^{2}$ So far, Khan et al and Silva et al mentioned five neonates with $C$. haemulonii candidemia (Table 1), ,3 and in two of the cases, the patient passed away. We reported the first two neonates with $C$. haemulonii candidemia in China treated with fluconazole and reviewed the 5 previously reported cases with this infection in the literature.

a. Department of Pediatrics, The Second Affiliated Hospital \& Yuying Children's Hospital of Wenzhou Medical University, Wenzhou, China

E-mail address:

Yong-Hai Zhou: zhouyonghai311@126.com

Funding: None.

Conflict of interest: None.

Received: 12-11-2017

Accepted: 3-26-2018

\section{CASE PRESENTATION}

Case 1

A 12-day-old-boy presented at our neonate intensive care unit from a township hospital with a history of abdominal distension for two days. This male baby was born at $30+5$ weeks gestation because of premature rupture of membranes by vaginal delivery and weighed $1520 \mathrm{~g}$ with Apgar scores of 5 at $1 \mathrm{~min}$ and 9 at $5 \mathrm{~min}$, respectively. The parturient is a 23 year old unemployed woman with normal history of prenatal examination. The premature baby was placed on tracheal intubation and mechanical ventilation at birth for the neonatal respiratory distress syndrome. After seven days the mechanical ventilation was stopped and the patient was put on nasal continuous positive airway pressure ventilation. The patient was fed by nasogastric tube and was thriving well. At the age of 10 days, he presented with abdominal distension and food residue. His X-ray displayed the pneumoperitoneum and C-reactive protein (CRP) was increased to $146(\mathrm{mg} / \mathrm{L})$. Therefore the baby was admitted to our unit. Soon after, the baby went into respiratory failure and required mechanical ventilation. Neonatal necrotizing enterocolitis (NEC) was subsequently confirmed. An emergency operation was carried out and a $40 \mathrm{~cm}$ long necrotic small intestine was removed. The patient was then started on meropenem (60 mg mg/ $\mathrm{kg} / \mathrm{d}$, divided every $8 \mathrm{~h}$ )

TABLE 1. Cases of neonatal C. haemulonii candidemia

\begin{tabular}{lcccc} 
NO. & Sex/age & $\begin{array}{c}\text { Country/ } \\
\text { region }\end{array}$ & Outcome & $\begin{array}{c}\text { Year of } \\
\text { publication } \\
\text { [Reference] }\end{array}$ \\
\hline 1 & $\mathrm{M} / 35$ weeks $(\mathrm{w})$ & Kuwait & Death & $2007[3]$ \\
2 & $\mathrm{M} / 26 \mathrm{w}$ & Kuwait & Survival & $2007[3]$ \\
3 & $\mathrm{~F} / 25 \mathrm{w}$ & Kuwait & Survival & $2007[3]$ \\
4 & $\mathrm{~F} / 31 \mathrm{w}$ & Kuwait & Death & $2007[3]$ \\
5 & $\mathrm{~F} / 26 \mathrm{w}$ & Brazil & Survival & $2015[4]$ \\
6 Case 1 & $\mathrm{M} / 30+5 \mathrm{w}$ & China & Survival & Our \\
7 Case 2 & $\mathrm{F} / 39+5 \mathrm{w}$ & China & Survival & Our \\
\hline
\end{tabular}


intravenously and parenteral nutrition were given by peripherally inserted central catheter (PICC). On the fifth day of hospitalization in our unit, an ascitic fluid culture revealed Pseudomonas aeruginosa and Aerobacter cloacae but blood culture was negative and the temperature was normal. Mechanical ventilation was stopped on the sixth day of hospitalization and CRP decreased to normal on the twenty-ninth day of hospitalization in our unit.

On the thirty-sixth day of hospitalization in our unit, the temperature of the baby rose to $38.4{ }^{\circ} \mathrm{C}$. Laboratory findings revealed a hemoglobin concentration of $95(\mathrm{~g} / \mathrm{L})$, a white blood cell count of $8.4 \times 109 / \mathrm{L}$ (60.1\% neutrophil), and a platelet count of $76 \times 109 / \mathrm{L}$. CRP was increased to $33 \mathrm{mg} / \mathrm{L}$. The PICC was removed. Blood and catheter tip samples were collected for culture.

After $72 \mathrm{~h}$, cultures of both blood and catheter tip produced several clonal formation units of yeast growth, which was subsequently identified as C. haemulonii by the Vitek 2 Compact Yeast cards (bioMérieux, France). The identity of C. haemulonii was confirmed by molecular biology methods. 3 The antifungal susceptibility testing showed that Candida isolate was susceptible to amphotericin B, ketoconazole, fluconazole, itraconazole, clotrimazole and nystatin. Fluconazole was administered $6(\mathrm{mg} / \mathrm{kg} / \mathrm{d})$ intravenously. After 30 days of fluconazole treatment, the infection was controlled and the baby recovered. On the eighty-eighth day of hospitalization, he was discharged when he could digest $50 \mathrm{ml}$ milk q $3 \mathrm{~h}$. In the subsequent followup, this baby grew well.

\section{Case 2}

A newborn with shortness of breath and III degrees meconium stained amniotic fluid presented to the neonatal intensive care unit. This female baby was born at $39+5$ weeks gestation by cesarean section for fetal distress and weighed $2650 \mathrm{~g}$ with Apgar scores of 6 at $1 \mathrm{~min}$ and 8 at $5 \mathrm{~min}$, respectively. The parturient is a 27 year old sales person and the prior prenatal examination history was normal. The baby was on mechanical ventilation and parenteral nutrition was given by umbilical venous catheter.

On the eleventh day of hospitalization, her body temperature rose to $38.4^{\circ} \mathrm{C}$. Laboratory findings revealed a hemoglobin concentration of $169 \mathrm{~g} / \mathrm{L}$, a white blood cell count of $15.7 \times 109 / \mathrm{L}$ (57.9\% neutrophil), and a platelet count of
$39 \times 109 / \mathrm{L}$. CRP was increased to $17(\mathrm{mg} / \mathrm{L})$. Therefore, the umbilical venous catheter was removed. Cultures of both blood and catheter tip produced some clonal formation units of yeast growth, which was subsequently identified as C. haemulonii by the Vitek 2 Compact Yeast cards (bioMérieux, France). The identity of $C$. haemulonii was confirmed by molecular biology methods. ${ }^{3}$ The antifungal susceptibility testing showed that Candida isolate was resistant to clotrimazole and nystatin, but susceptible to amphotericin B, ketoconazole, fluconazole and itraconazole. Fluconazole was administered $6(\mathrm{mg} / \mathrm{kg} / \mathrm{d})$ intravenously. The mechanical ventilation was removed on the sixteenth day of hospitalization. After 26 days of fluconazole treatment, the patient recovered. On the thirty-eighth day of hospitalization, this baby was discharged after two repeat blood culture results were negative. In the subsequent follow-up, the baby grew well.

\section{DISCUSSION}

In the past years, Candida species have been found to be important emerging nosocomial pathogens. Candida haemulonii, a kind of Candida species, has been reported to cause fungaemia, ${ }^{3-11}$ osteitis, ${ }^{12}$ peritonitis ${ }^{13}$ and chronic otitis media. 8 Up to now, the $C$. haemulonii candidemia has been described in five neonates, two $(40.0 \%)$ of whom passed away. The relevant features of the 5 reported cases with candidemia caused by $C$. haemulonii are summarized in the Table 1. Our descriptions of two neonates suffering from $C$. haemulonii candidemia are the first two cases in China.

Fever is the most common first sign. It is difficult to diagnose the $C$. haemulonii infection at the early stage by the nonspecific symptoms and signs. It is to be noted that thrombocytopenia was detected in both our cases which may be an indication of $C$. haemulonii candidemia.

According to the past reports, the possible risk factors of $C$. haemulonii candidemia are the widespread use of broad-spectrum antimicrobial agents, 6 the use of invasive therapeutic devices (eg. catheters and mechanical ventilation), ${ }^{6-10}$ premature patients, ${ }^{3,4}$ oncology patients, ${ }^{4,7,9,11}$ new surgical techniques ${ }^{5}$ and chronic kidney disease. ${ }^{9}$

In this report, Case 1, a premature who underwent an operation of NEC and who was on broad-spectrum antimicrobial agents. The baby was on mechanical ventilation and PICC support. The period of hospitalization in NICU was long. All these are risk factors of $C$. haemulonii 
candidemia in this baby. In spite of being a term infant, Case 2 also had risk factors of $C$. haemulonii candidemia; patient was on mechanical ventilation and had an umbilical venous catheter. Long-term catheter for hyperalimentation was the associated risk factor for candidemia, and the positive culture of the catheter tips suggested the possibility of catheter-related candidemia.

Amphotericin B, ketoconazole, fluconazole, itraconazole and clotrimazole are the common available agents for the treatment of severe fungal infections. However, C. haemulonii has been reported to be resistant to amphotericin $\mathrm{B}, 3,4,6,11$ fluconazole, ${ }^{3,7-10}$ itraconazole $^{3,7,8}$ flucytosine $^{11}$ and micafungin. ${ }^{5} \mathrm{Zia}$ et al. described four cases in which the candida isolates were all resistant to amphotericin B, fluconazole, and itraconazole and two of them passed away. ${ }^{3}$ In our study, the patients were treated with fluconazole and recovered. Both Candida isolates from our cases were susceptible to amphotericin B and fluconazole. From these results, we suspected that the multi-drug resistance, especially the resistance to amphotericin B, fluconazole, and itraconazole may be one of the important causes of death in C. haemulonii candidemia patients. The resistance of $C$. haemulonii represents a challenge in the treatment of candidiasis.

In conclusion, the candidemia caused by $C$. haemulonii is extremely rare but fatal in NICU. The clinical manifestations of $C$. haemulonii candidemia in the early stage are similar to those of common infection. From our findings, when fever and thrombocytopenia is detected in a neonate who has some underlying disease or invasive therapeutic devices (eg. PICC or umbilical venous catheter), the $C$. haemulonii candidemia should be suspected. The resistance of $C$. haemulonii represents a challenge in the treatment. It is the key point that the sensitive antifungal agents should be given and the existing PICC or umbilical venous catheter should be removed at the early stage.

\section{REFERENCES}

1. Lavarde V, Daniel F, Saez H, et al. Peritonite mycosique a Torulopsis haemulonii. Bul Soc Fr Mycol Med 1984; 13:173-6.

2. Panackal AA. Optimizing containment and control of Candida parapsilosis fungemia among neonates in the outbreak setting using a mathematical modeling approach. J Mycol 2013; 2013:413874.

3. Khan ZU, Al-Sweih NA, Ahmad S, et al. Outbreak of fungemia among neonates caused by Candida haemulonii resistant to amphotericin B, itraconazole, and fluconazole. J Clin Microbiol 2007; 45(6):2025-7.

4. Silva CM, Carvalho-Parahym AM, Macêdo DP, et al. Neonatal Candidemia Caused by Candida haemulonii Case Report and Review of Literature. Mycopathologia 2015; 180(1-2):69-73.

5. Muro MD, Motta Fde A, Burger M, et al. Echinocandin resistance in two Candida haemulonii isolates from pediatric patients. J Clin Microbiol 2012; 50(11):3783-5.

6. Rodero L, Cuenca-Estrella M, Córdoba S, et al. Transient fungemia caused by an amphotericin B-resistant isolate of Candida haemulonii. J Clin Microbiol 2002; 40(6):2266-9.

7. Giusiano G, Mangiaterra M, Garcia Saito V, et al. Fluconazole and itraconazole resistance of yeasts isolated from the bloodstream and catheters of hospitalized pediatric patients. Chemotherapy 2006; 52(5):254-9.

8. Kim MN, Shin JH, Sung H, et al. Candida haemulonii and closely related species at 5 university hospitals in Korea identification, antifungal susceptibility, and clinical features. Clin Infect Dis 2009; 48(6):e57-61.

9. RuanSY, Kuo YW, Huang CT, et al. Infections due to Candida haemulonii species identification, antifungal susceptibility and outcomes. Int J Antimicrob Agents 2010; 35(1):85-8.

10. Kim S, Ko KS, Moon SY, et al. Catheter-related candidemia caused by Candida haemulonii in a patient in long-term hospital care. J Korean Med Sci 2011; 26(2):297-300.

11. Almeida JN Jr, Motta AL, Rossi F, et al. First report of a clinical isolate of Candida haemulonii in Brazil. Clinics (Sao Paulo) 2012; 67(10):1229-31.

12. Crouzet J, Sotto A, Picard E, et al. A case of Candidahaemulonii osteitis clinical features, biochemical characteristics, and antifungal resistance profile. Clin Microbiol Infect 2011; 17(7):1068-70.

13. Yuvaraj A, Rohit A, Koshy PJ, et al. Rare occurrence of fatal Candida haemulonii peritonitis in a diabetic CAPD patient. Ren Fail 2014; 36(9):1466-7. 